\title{
Interaction parameters for the uptake of sulfur mustard mimics into polyurethane films
}

\author{
Tyler G. Grissom, Justin M. Sirrine, Timothy E. Long, Alan R. Esker, and John R. Morris* \\ Department of Chemistry, Virginia Tech, Blacksburg, Virginia 24061, United States
}

\begin{abstract}
Chemical warfare agent (CWA) sorption into polymeric coatings is an important consideration for preventing human exposure to CWAs. The specific nature of CWA-polymer interactions greatly influences the degree of gas uptake into a polymeric coating. By uncovering the role the various functional groups in CWAs play during gas sorption, better films and coatings can be engineered. This study utilized a quartz crystal microbalance to measure sulfur mustard (HD) analog (simulant) and hexane vapor uptake into polyurethane films. Analysis of the uptake data with Flory-Huggins theory yielded interaction parameter values $(\chi)$ between 0.24 and 0.40 for each HD simulant-polyurethane system compared to 0.97 for hexane, revealing the importance of the chlorine and sulfur groups in gas uptake at the thin film interface. We predict similar uptake behavior for HD in polyurethane coatings due to structural similarities between HD and the simulants.
\end{abstract}

Key words: Gas Uptake; Sulfur Mustard; Polyurethane; Film; Quartz Crystal Microbalance (QCM); Flory-Huggins Interaction Parameter 


\section{Introduction}

The chemical warfare agent (CWA) bis(2-chloroethyl)sulfide (sulfur mustard, HD) readily infiltrates polymeric coatings and other materials which prolongs the risk of human exposure. ${ }^{1-4}$ Willis et al. ${ }^{1}$ found that HD slowly off-gasses from polymeric coatings into the environment, further increasing the potential for human exposure. The presence of chlorine and sulfur groups in HD suggest HD is capable of having strong dipole and dispersive interactions with many polymeric coatings, which may facilitate gas uptake into the polymer. To date, little is understood about the relationship between CWA structure and polymeric coating properties that affect initial uptake, diffusion into the material, and residence times.

The work presented here utilized a quartz crystal microbalance with dissipation monitoring (QCM-D) to measure gas uptake of HD chemical analogs into polyurethanes, commonly used coating materials. Gas uptake measurements of less toxic, but structurally similar compounds to $\mathrm{HD}$, referred to as simulants, provided the advantage of being able to systematically probe the effect specific functional groups have on gas uptake. In addition to studying HD simulants, the commonly used solvent, hexane was also examined. While QCM-D gas uptake experiments involving chemical warfare agents and other volatile organic compounds are not novel, QCM-D use has seen limited application in probing fundamental interactions in gas-polymer systems. ${ }^{5-8}$

\section{Experimental Methods}

Abbreviations. CWA, chemical warfare agent; HD, sulfur mustard or mustard gas; QCM-D, quartz crystal microbalance with dissipation monitoring; 2-CEES, 2-chloroethyl ethyl sulfide; PTMO, poly(tetramethylene oxide); HMDI, Bis(4 isocyanatocyclohexyl)methane; BDO, 1,4butanediol; ${ }^{1} \mathrm{NMR}$, proton nuclear magnetic resonance; DSC, differential scanning calorimetry; TGA, thermogravimetric analysis 


\subsection{Materials}

Poly(tetramethylene oxide) (PTMO) oligomer (Terathane, Du Pont) with a number average molecular weight of $2000 \mathrm{~g} / \mathrm{mol}$ and 1,4-butanediol were purchased from Aldrich and dried in vacuo at $40{ }^{\circ} \mathrm{C}$ for $24 \mathrm{~h}$ prior to use. $\operatorname{Bis}(4$ isocyanatocyclohexyl)methane (HMDI) was graciously donated by Covestro with purity greater than $99.5 \%$. Chloroform $\left(\mathrm{CHCl}_{3}\right.$, Fisher Scientific, HPLC grade), tetrahydrofuran (THF, EMD Science, HPLC grade), and diethyl ether (Fisher Scientific, 99.9\%, anhydrous) were used as received. N,N-Dimethylformamide (DMF, Fisher Scientific, HPLC grade) was dried with a PureSolv ${ }^{\circledR}$ solvent purification system prior to use. Dibutyltindilaurate (DBTDL, 99\%) was dissolved in THF as a 1 wt \% solution. Sulfur mustard simulants: 1-chloropropane (99\%), 1-chlorobutane (anhydrous, 99.5\%), 1-chloropentane (99\%), 1,5-dicholoropentane (99\%), diethyl sulfide (98\%), 2-chloroethyl ethyl sulfide (98\%), and hexane (Spectrum, HPLC grade) were purchased from Sigma Aldrich and unless otherwise noted used as received. Gold (QSX-301) QCM-D sensors were purchased from Q-sense AB. The gold sensors are $14 \mathrm{~mm}$ in diameter with a $0.3 \mathrm{~mm}$ quartz thickness. The quartz is plated with a thin layer of chromium $(\sim 2 \mathrm{~nm})$, followed by a thin layer of gold $(\sim 100 \mathrm{~nm})$.

\subsection{Synthesis of 43 wt \% Hard Segment Content Polyurethane}

The model polymeric coating examined in this work was a segmented polyurethane prepared from a 2,000 g/mol poly(tetramethylene oxide) (PTMO), hydrogenated methylene diphenyl diisocyanate (HMDI), and 1,4-butanediol (BDO) with $43 \mathrm{wt} \%$ hard segments. The synthesis was adapted from Williams et al. ${ }^{9}$ Briefly, HMDI (66.8 mmol, $\left.17.536 \mathrm{~g}\right)$ was added dropwise to the reaction flask containing PTMO (15.0 mmol, $29.990 \mathrm{~g})$ and $50 \mathrm{ppm}$ DBTDL at $80^{\circ} \mathrm{C}$. Dry DMF $(100 \mathrm{~mL})$ was then introduced, followed with dropwise addition of 1,4-butanediol (51.8 mmol, 
$4.672 \mathrm{~g}$ ) which was allowed to react for $24 \mathrm{~h}$. The resulting reaction solution was twice purified via precipitation into diethyl ether. The resulting white, elastomeric product was subsequently dried in vacuo at $50{ }^{\circ} \mathrm{C}$ for $12 \mathrm{~h}$.

\subsection{Preparation of Polyurethane Films}

QCM-D sensors were cleaned prior to use by exposure to UV/ozone for 45 min (BioForce Nanoscience Inc. ProCleanerTM110), immersion into a 1:1:5 v/v solution of ammonium hydroxide/hydrogen peroxide/ultrapure water at $80{ }^{\circ} \mathrm{C}$ for $2 \mathrm{~h}$, followed by a rinse with ultrapure water and drying with nitrogen. The polyurethane was dissolved in chloroform to make $0.5-1.0$ wt $\%$ polymer solutions. The polyurethane solution was spin-coated onto the top of the cleaned QCM-D sensors at a spinning speed of $2000 \mathrm{rpm}$ for $1 \mathrm{~min}$. The bottom of each crystal was cleaned with acetone for the removal of residual polymeric material.

\subsection{Polyurethane Characterization}

Proton nuclear magnetic resonance $\left({ }^{1} \mathrm{H}\right.$ NMR) spectroscopy was performed in a Varian 400 $\mathrm{MHz}$ spectrometer in $\mathrm{CDCl}_{3}$ at $23{ }^{\circ} \mathrm{C}$. The polyurethane sample was stored in a desiccator after synthesis and was dried in vacuo at $50{ }^{\circ} \mathrm{C}$ for $12 \mathrm{~h}$ before analysis. Differential scanning calorimetry (DSC) was accomplished using a TA Instruments DSC Q1000 under a $\mathrm{N}_{2}$ atmosphere with a heat/cool/heat cycle performed at $10{ }^{\circ} \mathrm{C} \mathrm{min}^{-1}$. Prior to performing DSC, the sample was annealed in vacuo at $100{ }^{\circ} \mathrm{C}$ for $12 \mathrm{~h}$. Thermogravimetric analysis (TGA) was conducted with a TA Instruments TGA Q500 under $\mathrm{N}_{2}$ from $25^{\circ} \mathrm{C}$ to $600{ }^{\circ} \mathrm{C}$ at a heating rate of $10{ }^{\circ} \mathrm{C} \min ^{-1}$. Characterization results can be found in Supporting Information.

\subsection{QCM-D Sorption Experiments}


In order to measure gas uptake into the polyurethane films, the vapor of interest was entrained in nitrogen gas bubbled through a gas saturator cell. The polyurethane films were continuously exposed to the vapor of interest inside flow cell modules of a Q-Sense E4 QCM-D system, where changes in resonant frequencies and dissipation of the oscillating polyurethane-coated quartz crystals were measured. Vapor exposure continued until maximum gas uptake was achieved and the system reached equilibrium. Gas uptake was measured at controlled activity shown by Equation 1:

$$
a_{1}=\frac{p_{1}}{p_{1}^{*}}
$$

where $p_{1}$ is the vapor pressure of the simulant or solvent gas flowing above the sensor and $p_{1} *$ is the vapor pressure of the pure condensed vapor at the temperature of the polymer film. Activity was controlled over the range of 0.1 to 0.9 by independently varying the temperatures of the gas saturator cell and the coated quartz crystal (Table S1). See Supporting Information for a more detailed description of the QCM-D method.

\section{Results and Discussion}

The measured changes in frequency at equilibrium during QCM-D sorption experiments were used in the Sauerbrey relationship (Equation 2) to calculate mass uptake of the chemical of interest in the polymeric films: ${ }^{10}$

$$
\frac{\Delta f}{n}=-C_{\mathrm{f}} \Delta m
$$

where $\Delta f$ is the change in resonant frequency after an applied load $(\mathrm{Hz}), C_{\mathrm{f}}$ is the sensitivity factor which is equal to $56.6 \mathrm{~Hz} \mathrm{~cm}^{2} \mu \mathrm{g}^{-1}, n$ is the overtone number, which can take on only odd integer values, and $\Delta m$ is the change in mass per unit area due to the applied load. As dissipation 
in these systems was negligible $\left(<0.5 \%\right.$ of $\left.\Delta \mathrm{f} / \mathrm{n} \times 10^{-6}\right)$ and the frequency values for multiple overtone values overlapped, criteria for use of the Sauerbrey equation were met.

Assuming additive volumes, measured changes in frequency values collected during gas exposure were used to calculate simulant and hexane volume fractions, $\phi_{1}$, at equilibrium in the swollen polyurethane films:

$$
\phi_{1}=\frac{\frac{\left(\Delta f_{\mathrm{S}} / n\right)}{\rho_{\mathrm{S}}}}{\frac{\left(\Delta f_{\mathrm{S}} / n\right)}{\rho_{\mathrm{S}}}+\frac{\left(\Delta f_{\mathrm{p}} / n\right)}{\rho_{\mathrm{p}}}}
$$

where $\Delta f_{\mathrm{s}}$ is the change in frequency due to simulant or hexane uptake, $\Delta f_{\mathrm{p}}$ is the change in frequency due to the polyurethane film, and $\rho_{\mathrm{s}}$ and $\rho_{\mathrm{p}}$ are the densities of the condensed simulant or hexane and polyurethane, respectively. Calculated volume fractions reveal each simulant and hexane readily penetrated and absorbed into the polyurethane films across the entire activity range studied. A swelling plot containing volume fraction versus activity is shown in Figure 1. All systems exhibit a rise in the volume fraction with increasing simulant activity, which indicates greater gas uptake into the polyurethane films at higher activities. At high activities, volume fraction values approach 0.5 for HD simulants, denoting a large degree of swelling of the polyurethane film. Gas uptake was not found to be significantly different between any of the HD simulants across the entire activity range; however, our results show notably lower swelling for hexane. 


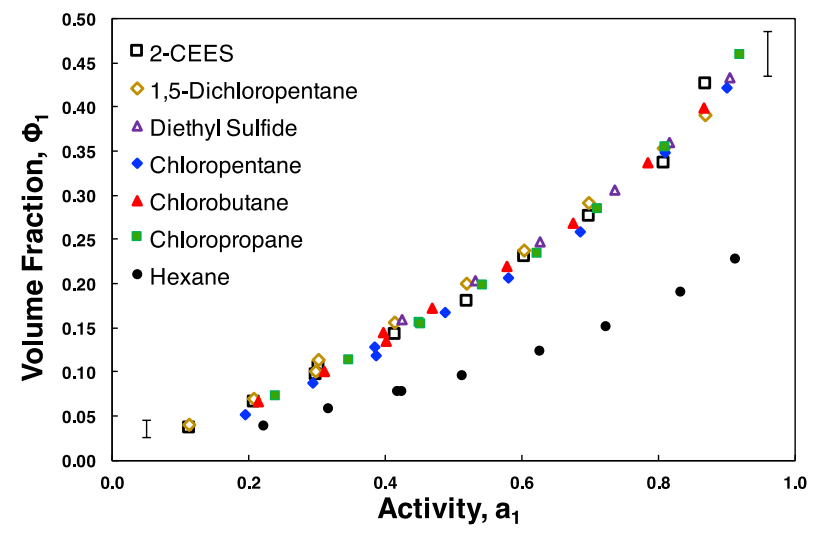

Figure 1. Gas uptake of HD simulants and hexane in polyurethane versus activity. Larger volume fraction, $\phi_{1}$, values correspond to greater uptake into the polyurethane. Error bars increase in size with increasing $a_{1}$ as indicated on the graph for the lowest and highest activities and signify \pm 1 standard deviation.

The nearly indistinguishable $\phi_{1}$ values between HD simulants as a function of $a_{1}$ provides insight into the role each functional group plays in gas uptake. For example, similar volume fraction values between chloropropane, chlorobutane, and chloropentane for a given $a_{1}$ signify that the additional dispersion forces from the methylene units have a limited effect on the overall polymer swelling within this chain length regime.

While the additional dispersive forces due to added methylene units in the n-chloroalkane simulants did not result in changes in $\phi_{1}$ vs $a_{1}$, replacement of the chlorine with a $\mathrm{CH}_{3}$ group, as is the case for hexane, reduced swelling by nearly $50 \%$. The lack of a highly electronegative chlorine atom in hexane results in a non-polar molecule capable of only weak dispersive interactions with the polyurethane film. The polar, chlorine containing simulants interact with the polymer through both dipole-dipole and dispersive interactions which enhance polymer swelling. Volume fractions for diethyl sulfide at similar activity matched the n-chloroalkanes, further supporting the idea that the ability to have dispersive and dipole-dipole interactions opposed to only weak dispersive interactions greatly increased the degree of swelling in these polymer films. 
The presence of a second chlorine or sulfur atom, found in 1,5-dichloropentane and 2chloroethyl ethyl sulfide, did not cause additional swelling compared to diethyl sulfide and the nchloroalkanes. The addition of a second electronegative group along the simulant chain decreases the dipole moment of the molecule, but increases its polarizability (Table 1), which suggests that the degree of polymer swelling is influenced by the strength of both the dipole moment and polarizability of the molecule.

Despite the differences in polarizability and dipole moment values of each of the HD simulants, we observed essentially identical $\phi_{1}$ vs $a_{1}$ plots (Figure 1) for sorption of each gas into the polyurethane films. The smaller uptake values observed from the similarly-sized hexane reveals the importance of the presence of chlorine and/or sulfur in gas uptake in the polyurethane films. Due to the similarity in chemical structures, polarities, and polarizabilities of the simulant molecules to the actual chemical agent, HD, we would expect HD volume fraction values in the polyurethane films to be very similar to those of the simulants.

Polymer-simulant interaction parameters for the data in Figure 1 were obtained from FloryHuggins theory: ${ }^{11-12}$

$$
\ln \left(a_{1}\right)=\ln \left(\frac{p_{1}}{p_{1}^{*}}\right)=\ln \left(1-\phi_{2}\right)+\phi_{2}+\chi \phi_{2}^{2}
$$

where $\phi_{2}$ is the volume fraction of the polymer $\left(\phi_{2}=1-\phi_{1}\right)$ and $\chi$ is the Flory-Huggins interaction parameter. Interaction parameters were calculated by solving for $\chi$ at each activity-volume fraction combination, then averaging the values together. Values of $\chi$ for each HD simulant were between 0.24 and 0.40, up to activity values of approximately 0.85 (Table 1, Figure S6) and did not exhibit statistically significant differences. Interaction parameters at activities greater than 0.85 showed a positive deviation due to the polymer cross-linking's resistance to additional 
swelling. Interaction parameters for hexane however were determined to be 0.97 , and showed only a limited volume fraction dependence within our experimental conditions, further supporting our claim that the effect of cross-linking only becomes significant under highswelling conditions. According to Flory-Huggins polymer solution theory, polymer-solvent systems with $\chi$ values less than 0.5 are classified as "good solvents", 13 while hexane, with a $\chi$ value greater than 0.5 , would be classified as a poor solvent. The values in Table 1 strongly suggest that the dipole moment afforded by the $\mathrm{S}$ and $\mathrm{Cl}$ atoms in the $\mathrm{HD}$ simulants relative to hexane are responsible for improved compatibility with the polyurethane. Considering the degree to which the chemical structures of the simulant molecules were altered relative to hexane, we predict a similar $\chi$ value for HD in the polyurethane $(\chi \sim 0.25$ to 0.4$)$.

Table 1. Flory-Huggins interaction parameters, dipole moments, and polarizability values for hexane, HD simulants, and HD. Calculations were conducted using B3LYOP/aug-cc-pVTZ and reasonably agree with literature values. Error bars of \pm 1 standard deviation for interaction parameters are reported.

\begin{tabular}{|c|c|c|c|c|c|}
\hline \multirow[b]{2}{*}{ Compound } & \multirow{2}{*}{$\begin{array}{c}\text { Interaction } \\
\text { Parameter, } \chi\end{array}$} & \multicolumn{2}{|c|}{ Dipole Moment, $\mu$ (D) } & \multicolumn{2}{|c|}{$\begin{array}{l}\text { Polarizability, } \alpha\left(\AA^{3}\right) \\
\text { Literature }^{1}\end{array}$} \\
\hline & & Literature & Calculated & $\underset{4}{\text { Literature }}$ & Calculated \\
\hline Hexane & $0.96 \pm 0.05$ & $0.000^{15}$ & 0.000 & 11.9 & 11.71 \\
\hline Chloropropane & $0.35 \pm 0.05$ & $2.051^{15}$ & 2.327 & 10.0 & 8.22 \\
\hline Chlorobutane & $0.31 \pm 0.06$ & $2.051^{15}$ & 2.424 & 11.3 & 10.10 \\
\hline Chloropentane & $0.39 \pm 0.05$ & $2.159^{15}$ & 2.443 & 12.0 & 12.01 \\
\hline Diethyl Sulfide & $0.33 \pm 0.07$ & $1.541^{15}$ & 1.708 & 10.8 & 11.18 \\
\hline 1,5-Dichloropentane & $0.24 \pm 0.11$ & $2.359^{\mathrm{a}, 15}$ & 1.992 & - & 14.23 \\
\hline 2-CEES & $0.31 \pm 0.05$ & $1.645^{16}$ & 2.091 & - & 13.51 \\
\hline HD & - & $0.78^{17}$ & 1.182 & - & 15.83 \\
\hline
\end{tabular}

${ }^{\mathrm{a}}$ Measured in benzene

\section{Conclusions}


To gain insight into how sulfur mustard interacts with polymeric materials, HD simulant sorption studies were carried out using a QCM-D method. Investigation of simulants with slight differences in chemical structures revealed the role the various functional groups on HD play in gas absorption, specifically the importance of chlorine and sulfur, which impart a permanent dipole on the molecule. We have shown that uptake of HD simulants into polyurethane films are greatly influenced by a combination of dipole moment strength and polarizability. Despite having a similar polarizability and size as the simulants, hexane exhibited lower uptake and as a result a much larger interaction parameter value than the simulants due to the absence of a permanent dipole moment.

Future work will examine how properties of the polyurethane film, such as varying hard:soft segment ratios or adding functionality to the polymer chains, affect gas uptake. Construction of structure-function relationships for both polymer and gas structures and the role they play in gas uptake, may provide insight into how chemical warfare agents, absorb, diffuse, and linger in polymeric coatings.

\section{Supporting Information}

Relevant experimental procedures, results, and data not included in the paper associated with polymer characterization including proton nuclear magnetic resonance ( ${ }^{1} \mathrm{H}$ NMR) spectroscopy, differential scanning calorimetry (DSC), and thermogravimetric analysis (TGA), as well as representative quartz crystal microbalance with dissipation monitoring (QCM-D) gas uptake data can be located in the Supporting Information.

\section{Author Information}

Corresponding Author 
*John R. Morris, E-mail: jrmorris@vt.edu

\section{Author Contributions}

The paper was written through contributions of all authors. All authors have given approval to the final version of the paper.

\section{Notes}

The authors declare no competing financial interest.

\section{Acknowledgments}

This research is based upon work supported by the Army Research Office (ARO) and the Defense Threat Reduction Agency via [W911NF-15-2-0107]. The views and conclusions contained herein are those of the authors and should not be interpreted as necessarily representing the official policies or endorsements, either expressed or implied, of the ARO, or the U.S. Government. The U.S. Government is authorized to reproduce and distribute reprints for Governmental purposes notwithstanding any copyright annotation thereon. 


\section{References}

(1) Willis, M. P.; Mantooth, B. A.; Lalain, T. A., Novel Methodology for the Estimation of Chemical Warfare Agent Mass Transport Dynamics. Part II: Absorption. J. Phys. Chem. C 2012, 116 (1), 546-554.

(2) Wagner, G. W.; MacIver, B. K., Degradation and Fate of Mustard in Soil as Determined by C-13 Mas Nmr. Langmuir 1998, 14 (24), 6930-6934.

(3) Brevett, C. A. S.; Sumpter, K. B.; Wagner, G. W.; Rice, J. S., Degradation of the Blister Agent Sulfur Mustard, Bis(2-Chloroethyl) Sulfide, on Concrete. J. Hazard. Mater. 2007, 140 (12), 353-360.

(4) Navaz, H. K.; Markicevic, B.; Zand, A. R.; Sikorski, Y.; Chan, E.; Sanders, M.;

D'Onofrio, T. G., Sessile Droplet Spread into Porous Substrates - Determination of Capillary Pressure Using a Continuum Approach. J. Colloid Interface Sci. 2008, 325 (2), 440-446.

(5) Price, G. J.; Haddon, D. A.; Bainbridge, A.; Buley, J. M., Vapour Sorption Studies of Polymer-Solution Thermodynamics Using a Piezoelectric Quartz Crystal Microbalance. Polym. Int. 2006, 55 (7), 816-824.

(6) Wibawa, G.; Khoiroh, I.; Afrizal, D.; Suki, G., Solubilities of Dichloromethane, Diethyl Ether, Ethyl Acetate, and Nitrobenzene in Three Polymers Using the Piezoelectric Quartz Sorption Method. J. Chem. Eng. Data 2010, 55 (12), 5581-5586.

(7) Wibawa, G.; Hatano, R.; Sato, Y.; Takishima, S.; Masuoka, H., Solubilities of 11 Polar Organic Solvents in Four Polymers Using the Piezoelectric-Quartz Sorption Method. J. Chem. Eng. Data 2002, 47 (4), 1022-1029.

(8) Du, X. S.; Wang, Z. D.; Huang, J.; Tao, S. L.; Tang, X. Z.; Jiang, Y. D., A New Polysiloxane Coating on Qcm Sensor for Dmmp Vapor Detection. J. Mater. Sci. 2009, 44 (21), 5872-5876.

(9) Williams, S. R.; Wang, W. Q.; Winey, K. I.; Long, T. E., Synthesis and Morphology of Segmented Poly(Tetramethylene Oxide)-Based Polyurethanes Containing Phosphonium Salts. Macromolecules 2008, 41 (23), 9072-9079.

(10) Sauerbrey, G., Verwendung Von Schwingquarzen Zur Wagung Dunner Schichten Und Zur Mikrowagung. Zeitschrift Fur Physik 1959, 155 (2), 206-222.

(11) Flory, P. I., Thermodynamics of High Polymer Solutions. J. Chem. Phys. 1942, 10 (1), 51-61.

(12) Huggins, M. L., Solutions of Long Chain Compounds. J. Chem. Phys. 1941, 9 (5), 440440.

(13) Flory, P. J., Principles of Polymer Chemistry. Cornell University Press: Ithaca, New York, 1953.

(14) Maryott, A. A.; Buckley, F., U. S. National Bureau of Standards Circular No. 5371953.

(15) Yaws, C. L., Thermophysical Properties of Chemicals and Hydrocarbons. William Andrew Publishing: Norwich, NY, 2009.

(16) Plusquellic, D. F.; Suenram, R. D.; Mate, B.; Jensen, J. O.; Samuels, A. C., The Conformational Structures and Dipole Moments of Ethyl Sulfide in the Gas Phase. J. Chem. Phys. 2001, 115 (7), 3057-3067.

(17) Politzer, P.; Habibollahzadeh, D., Bond-Breaking Energies for 2,2'-Dichlorodiethyl Sulfide (Sulfur Mustard) in Media of Different Dielectric-Constants. J. Phys. Chem. 1994, 98 (6), 1576-1578. 


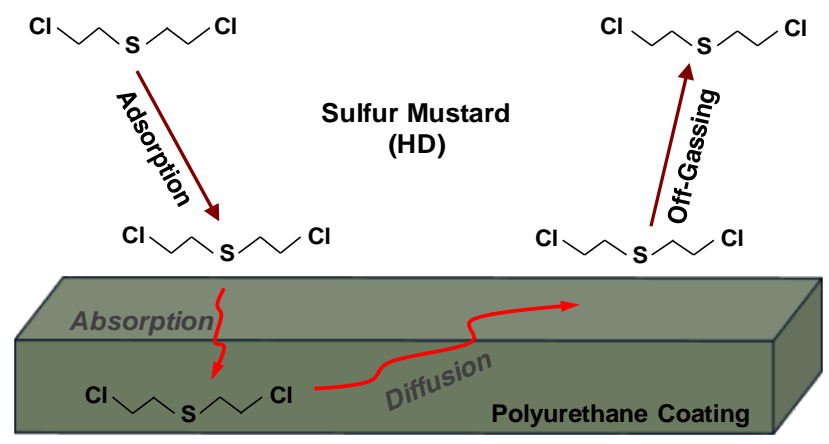

For Table of Contents and Abstract Only 